\title{
Damage Identification of Multi-span Bridge Structure Based on the Recognition of Influence Line
}

\author{
Zhang Yunkai, Xie Qingli, Li Guohua* and Ye Yuntao \\ Beijing University of Civil Engineering and Architecture,Beijing,102616, China
}

\begin{abstract}
The stress and deflection effects of the line changes before and after the bridge damage are used as indicators to evaluate the bridge damage and the initial damage site. Then a method of combining information is proposed to improve the accuracy of the damage site. Three-span continuous reinforced concrete was used in the analysis. According to the test, the effectiveness of damage identification based on the damage change of the influence line and the feasibility of the damage location method based on multisensory information fusion are confirmed.
\end{abstract}

\section{Introduction}

For damage identification and analysis of multi-span bridge structures, only numerical simulation or component testing can be carried out in the past, due to equipment limitations and economic conditions. However, it is difficult for numerical simulation to reflect the actual characteristics of components. Therefore, large-scale or full-scale seismic tests must be performed on large and complex structures to make up for the lack of theoretical analysis. Conventional test methods include artificial static test methods, earthquake simulation test methods for shaking tables, and artificial dynamics test methods ${ }^{[1]}$. With the upgrading and transformation of reconstruction laboratories around the world, modern seismic test methods are used for research. This article mainly introduces modern inspection methods, and explains their applications and prospects in a wide range of bridge structures.

\section{Method Introduction}

\subsection{Current Status of Structural Damage Detection}

As a kind of structure, the bridge will be damaged to some extent, due to the flaws in its design theory, the natural aging of materials and the corrosion in the external environment since its construction. When the damage reaches a certain level, cracks, structural deformation and even structural defects will appear, which will cause great harm to peoples' life safety and social and economic development. And once it happens, it will bring very serious consequences. In recent decades, domestic and

\footnotetext{
*Corresponding author's e-mail: liguohua@bucea.edu.cn
}

foreign researchers have proposed various methods to identify the bridge structure, so as to ascertain the damage of the bridge, mainly through the following three aspects. The first one is to identify whether there is damage ${ }^{[2]}$. The second one is to indicate the damage degree. And the third one is to determine the bearing capacity of the structure with known damage. The general methods after theoretical analysis and engineering verification are as follows:

\subsubsection{Identification Method of Structural Damage Based on Static Test}

This method measure the response of specific parts of the structure, such as static variables, displacement, load, and cracks, and compares the measured data with the original model analysis. Generally, under the condition of continuously correcting the deflection coefficient, the theoretical value obtained through the model is consistent with the actual value measured in the test. Although static recognition has high accuracy and stability, it places high requirements on the test conditions. Moreover, it is not easy to collect a large amount of data during the experiment.

\subsubsection{Structural Damage Identification Method Based on Dynamic Test}

This method maximizes the vibration measurement points at different points in the structure to be tested, collects vibration signals generated by the environment or load and gets the dynamic parameters of the structure. Then, the modal corresponds to physical variables, such as bending stiffness and structural quality, and the damage of the structure is analyzed. Compared with the fixed test 
method used to determine damage, this method reduces the workload and economic cost, but the structure loss recognition function based on the powerful test is greatly affected by noise. In addition, the current technical level can only collect limited data on site, and requires high test equipment, so there are many limitations.

\subsection{Structural Damage Identification Method Based on Deflection Influence Line}

When a concentrated load with a constant reference value (usually vertically downwards) moves along the structure, the graph representing the changing law of a certain value is called the effect line of that value. Kou Xiaona applied the curvature influence line (including its first derivative and second derivative) for the first time in the field of tissue recognition. The mechanical theory is used to numerically analyze the reinforced concrete simply supported beam model to obtain the deflection lines of the simply supported beam and its first derivative and second derivative $^{[3]}$. Then she uses ANSYS for modeling and analysis to check the damaged structure on the curvature line and the obvious characteristics of its first and second derivatives. Finally, through experiments, it is verified that the flexural effect line can identify the existence of damage, and at the same time, it can identify the extent of damage very weakly. However, this method is not sensitive to local damage and can't identify the location of the damage through the broken line. Curvature influence line is another effective method to identify damage, which can detect the existence of damage and judge its location and degree of damage ${ }^{[4]}$. However, the process of solving the second derivative of the bending effect line is very complicated and very sensitive to the external environment. The data has a major influence on temperature and noise. Kou Xiaona's research creatively proposed a new idea of using multiple influence lines to identify structures, but the research model is only supported by beams with the simplest structure, so it is not clear whether the line recognition effect is more suitable for complex continuous beam structures. Jia Yaping established a continuous beam bridge index to identify the structure of the deflection line. He also studied the differences between the shock lines, the first and second derivatives of the shock deflection difference and the second derivative of the deflection shock lines. Then, he uses the three-span model to verify these four indicators and observe whether they can indicate damage and the location of damage. However, the model he used in the experiment was an alloy wheel to simulate the connecting beam, but the actual bridge mainly uses concrete material (single material), with different performance of the two materials. The test only applies a fixed load without considering the heavy load in the actual situation, which greatly affected the accuracy. Sun Ke further developed a method for identifying damage to buildings based on the differences in the influence lines of symmetrical measurement points. Firstly, based on the energy law, the influence line formula of each point on the simply supported beam is obtained in the case of partial failure. In order to eliminate the error, the difference of the displacement effect line of the two symmetrical measuring points at the center of the range was checked under symmetrical load, which was used as an index to identify the local damage of the simply supported beam, and the value of the index was proved through numerical experiments. Without contracting the surface data, this method can easily obtain symmetric displacement index positions for local damage identification ${ }^{[5]}$. However, the damage to the symmetrical position can't be determined. Only relatively simple structures are discussed, while the applicable effects for large and complex structures are not clear. Xu Wentao deeply analyzed the influence of the random operation of the axle on the influence line of the bridge. He converted the uninterrupted random stimulus caused by the unevenness of the bridge surface into a shorter stimulus surface to obtain the arbitrary response of the bridge, and found that the speed of the vehicle and the inequality of the bridge surface have a great influence on the random response of the bridge. The response of the influence line to the vehicle speed is related to the parameters of the vehicle bridge (the excitation frequency of the vehicle to the bridge and the frequency of the bridge). But there is no way to eliminate errors. Xu Jian established an error index to strengthen the influence line when the bridge bends caused by vehicle noise and other factors $^{[7]}$. He analyzed and processed the wave changes in the directly accumulated curve process, and removed the wave coefficients of noise interference in each frequency band, thereby retaining the original signal, and then reconstructed the influence line. Experiments show that the method meets the actual engineering requirements in terms of accuracy and usability.

\subsection{Damage Identification Index Based on Changes of Influence Line}

The vertical force of the mobile unit acts on the bridge in an unbreakable state, and the bridge response can establish a base influence line and represent the unbreakable state of the bridge. If the bridge is damaged, the influence line may change accordingly after the bridge is used for a period of time. Compared with the base influence line, the amount of change contains damage information, which may reflect changes in the inherent characteristics of the bridge structure ${ }^{[6]}$. Therefore, the change of the influence line can be regarded as an identification indicator of loss, namely:

$$
\Omega(x)=\Phi(x)-\Phi_{B L}(x)
$$

In the formula, the influence line of the state to be checked and the reference (non-destructive) influence line are expressed by $\Phi(x)$ and $\Phi_{\mathrm{BL}}(x)$ as a function of the position along the bridge. If the structure is not damaged or the production site of the influence line is far away from the damage location, the change in the influence line will be very small and can be ignored. But the maximum value of the influence line derivative will change significantly, which can be used as the basis for detecting damage, so as to identify whether the bridge is damaged and the location of the damage. 


\subsection{Damage Location Based on Information Fusion of Multiple Influence Lines}

Generally, bridge health monitoring or safety inspection will select multiple key locations to arrange sensors in order to obtain recognition results and change the indications of influence line at multiple locations. If the damage change is based on information about a single sensor, the result will be greatly affected by arbitrary factors such as measurement noise. Therefore, it is planned to improve the consistency information (such as damage) of multiple signals by merging various location information and eliminate inconsistent information (such as noise) to improve the susceptibility to damage and the credibility of decision-making ${ }^{[8]}$. If two locations are to be viewed, $m=\{1,2,3 \ldots, m\}$, and the information of one sensor is analyzed, the probability distribution function of the variance of the influence line changes can be defined as:

$$
m=\left[\begin{array}{cccc}
m_{1}\left(x_{1}\right) & m_{1}\left(x_{2}\right) & \ldots & m_{1}\left(x_{m}\right) \\
m_{2}\left(x_{1}\right) & m_{2}\left(x_{2}\right) & \ldots & m_{2}\left(x_{m}\right) \\
\ldots & \ldots & m_{i}\left(x_{j}\right) & \ldots \\
m_{n}\left(x_{1}\right) & m_{n}\left(x_{2}\right) & \ldots & m_{n}\left(x_{m}\right)
\end{array}\right]
$$

Among them, $m_{i}\left(x_{i}\right)$ represents the probability distribution function used to determine whether the second location to be inspected is damaged by changing the influence of the sensor:

$$
m_{i}\left(x_{i}\right)=\frac{\left|\Omega_{i}\left(x_{i}\right)\right|}{\sum_{j=1}^{m}\left|\Omega_{i}\left(x_{i}\right)\right|}
$$

\section{Numerical Simulation Analysis}

\subsection{Introduction of Software Analysis}

In the experiment, the wide continuous girder bridge is simplified as a bridge with three continuous girder spans, which is designed as a rectangle.

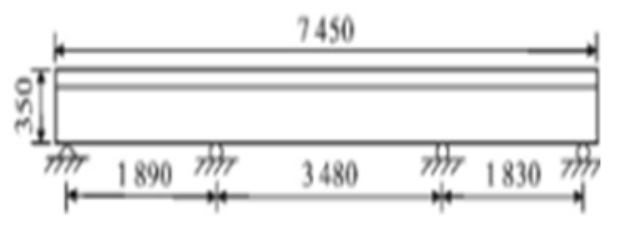

Figure 1 Dimension of reinforced concrete three-span continuous beam structure position: $\mathrm{mm}$

At $1 / 4,3 / 8,1 / 2,5 / 8,3 / 4$ (S1, S2, S3, S4, S5) of the continuous beam, five load meters and five displacement meters are used to collect the load and deflection response of the beam during the driving of the trolley, and then determine the influence of the load line and deflection on these positions.

\subsection{Verification of Damage Detection Method}

In the failure state, strain gauges are arranged in 5 different positions to collect various data, according to the load response before and after the failure, The result is shown in figure 2.

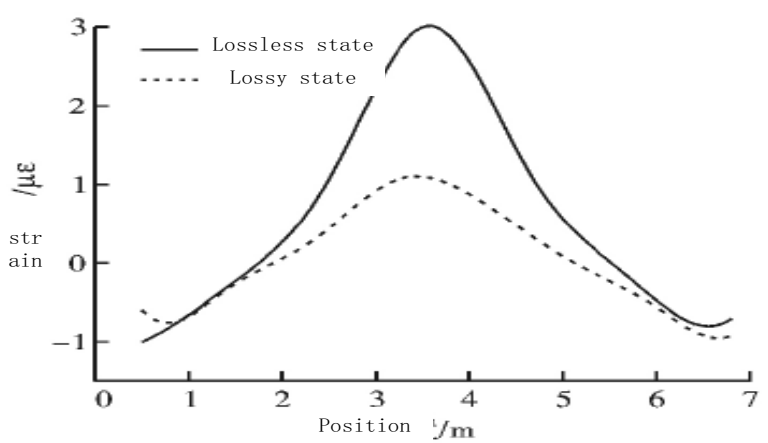

(a) Strain influence line

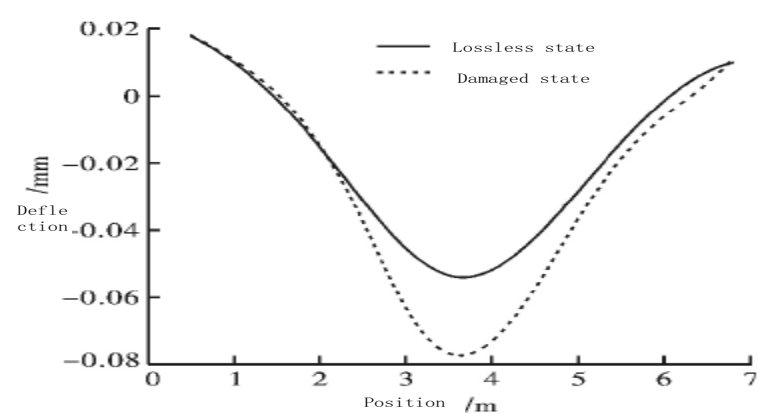

(b) Deflection influence line

Figure 2 Recognition results of influence lines before and after continuous beam damage

In order to study the change of the influence line due to slight damage, the effect index of the change limit can be calculated from the influence line before and after the damage by using formula (1). First of all, the change curves of the five curvature effect lines in Figure 3(b) show that all curves have obvious fluctuations, with close maximum position to the elbow and everything fallen within the damaged area. This shows that the obvious and regular oscillation of the curve can be used as an indicator of damage detection, and the maximum value of the curve can initially determine the location of the damage. The load effect change index at the three positions of the medium voltage $1 / 2$ span, $3 / 8$ span and $5 / 8$ span showed greater fluctuations than 1 .

The influence line changes of $1 / 4$ span and 3/4 span are difficult to judge whether they are damaged, with only the position of $1 / 2$ span affected and the maximum noise line changes just in the damaged area, which can be explained as the load is more sensitive to local damage, with only in a small area near the damage. 


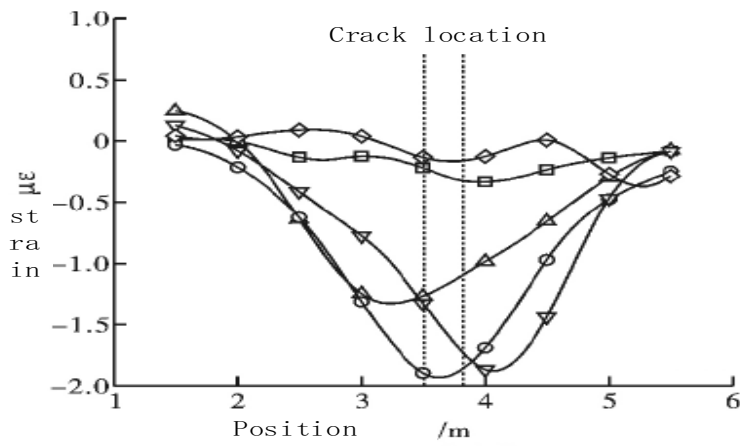

(a)Strain influence line

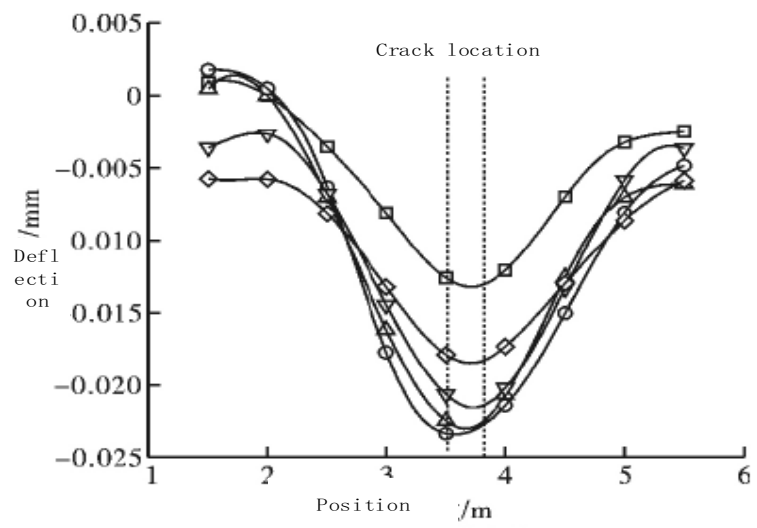

(b)Deflection influence line

Figure 3 the change index of influence line of different measuring points

It can be seen from the results of the stress influence line in Figure 3(a) that if damage is diagnosed based only on the influence line of a certain measuring point, the results obtained from different measuring points may be contradictory, thereby reducing the reliability of damage analysis result. The multi-line merging method for damaged locations is proposed. According to the change of the influence line before and after continuous radiation damage, the formula to calculate each probability distribution function is used and the probability distribution matrix of the formula is constructed. Using multi-sensory information fusion, a formula based on distance weight coefficients can be used to calculate the probability distribution function of each location to be viewed. According to the information fusion on different numbers of sensors, the influence of the damage location is investigated and compared, which is shown in Figure 3. It can be clearly seen that as the number of sensors increases, the probability distribution function near the damaged part gradually increases in the middle. After combining the information on 3 or 5 sensors, the difference between the probability distribution function near the damaged location and the damaged location is more important, with the probability distribution function of the location far away from the damaged location at basically zero. In addition, different types of sensors are used to combine information to verify the feasibility of locating damage. The results of the damage analysis show that different types of sensors can perform information merging, which may cause location damage. All in all, the following conclusions can be drawn that the many effects of merged line information can actually increase the steady flow of information in labels from many sources (such as organizational changes caused by damage), and eliminate inconsistencies and noise figures) to increase the sensitivity and damage effects of damage.

On the basis of frequency analysis, the analysis of static control is carried out. According to the number of nodes, the lower string is divided into 11 , and the nodes are numbered $1,2,3,4,5,6,7,8,9,10,11$ from left to right in the model, which is loaded according to the specifications of the bridge model. Since the final component software is in an ideal state of imitation, the flexibility and displacement of the bridge structure are the same under the same loading and unloading conditions. Therefore, this study only performs charging process analysis. The deformation diagram below shows when fully charged under different working conditions and the displacement diagram of each measuring point. The working condition diagram is shown in Figure 4-8.

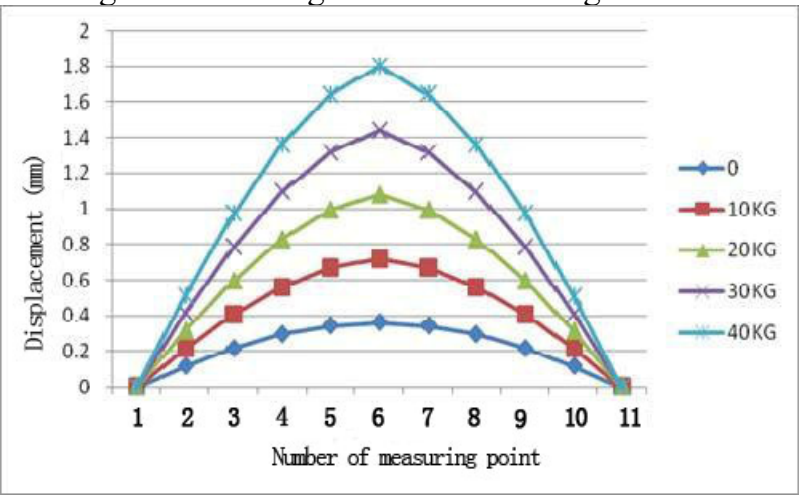

Figure 4 the first working condition one

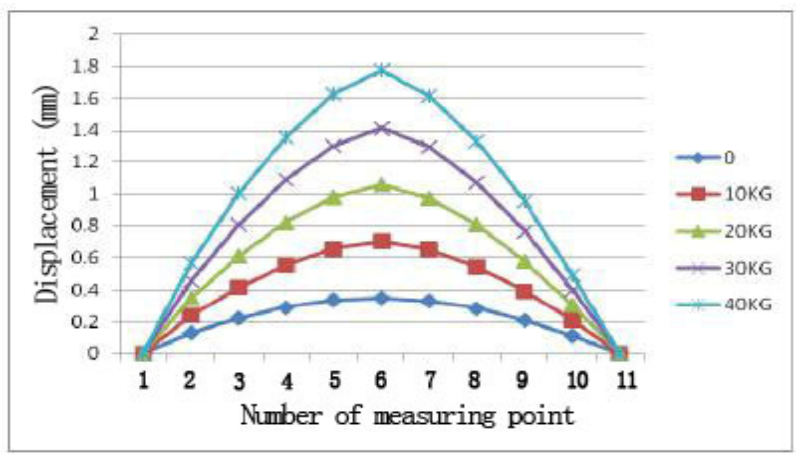

Figure 5 the second working condition

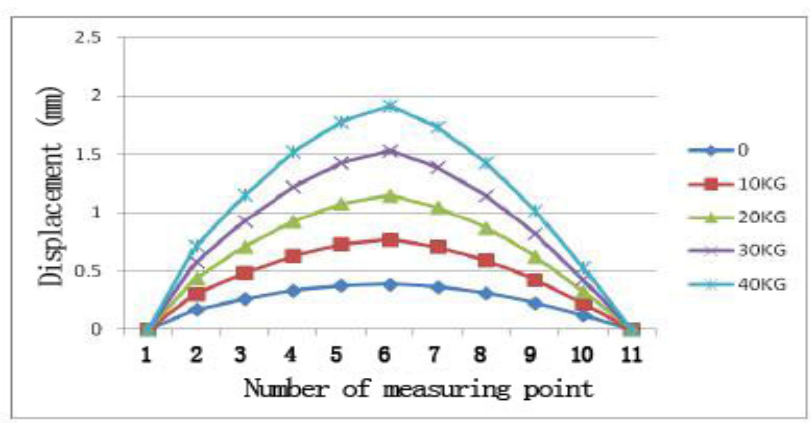

Figure 6 the third working condition 


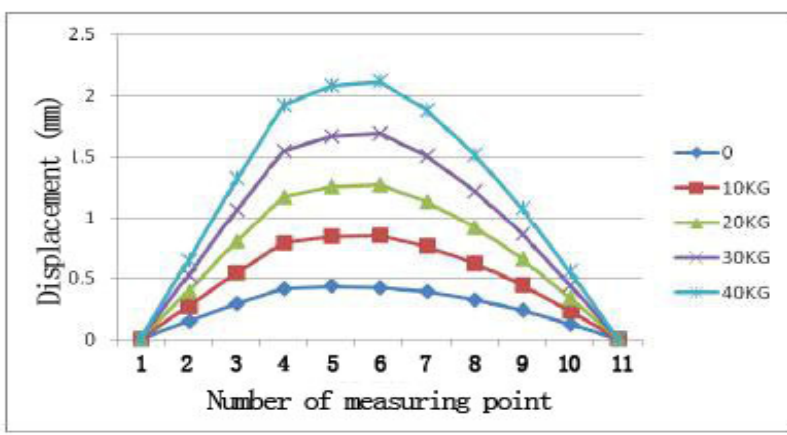

Figure7 the fourth working condition

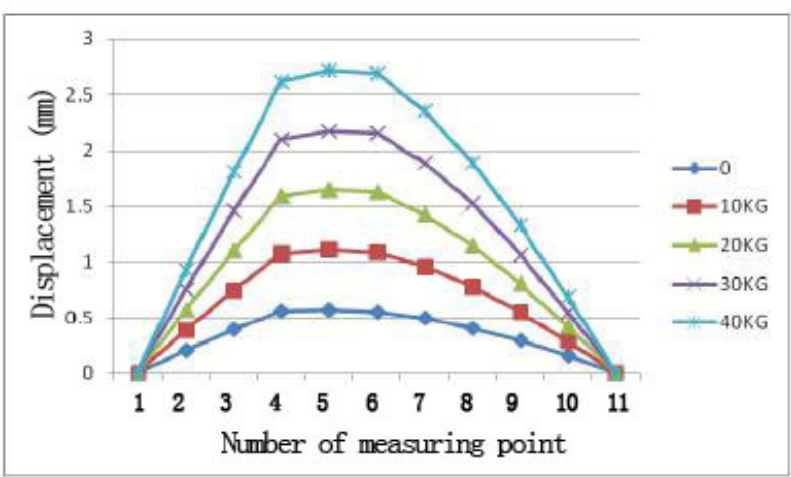

Figure 8 the fifth working condition

It can be seen from the displacement trajectory diagram of the above damage condition that in the charging process of each damage condition, all conditions are asymmetric except for the complete development of a complete damage (no damage) under the displacement curve condition. When the graph assumes that the damage is large and asymmetric, the displacement near the damaged part will fluctuate greatly or the largest, or the displacement change at the measurement point 5 will be the largest. Under other working conditions, the displacement change in the middle of the span of measuring point 6 is the largest, while that of measurement point 2 and measurement point 10 are the smallest. Measurement point 1 and measurement point 11 are limited by the simple beam end, so the displacement is zero. The maximum displacement of each measuring point under its own weight and full load is shown in the table above. The maximum displacement is the displacement of the central voltage measurement point under ten operating conditions, which is $7.76 \mathrm{~mm}$.

\section{Conclusion}

Changes in load and curvature will affect the influence lines before and after the damage of the bridge to identify whether the bridge is damaged and the location of the damage. A multi-sensory information fusion method is proposed to improve the accuracy of the damage site. Finally, by testing the continuous beam, the verification value is based on the feasibility of the impact line and the damaged part.

\section{References}

1. Wu Guifei, Zhang Yanqing, Sun Ke. Research on Bridge Damage Identification Based on Displacement Influence Lines of Multiple Measuring Points [J]. Journal of China Three Gorges University (Natural Science Edition), 2018, v.40; No. 161(02): 59-64.

2. Ning Tingting, Li Dongsheng. Truss damage identification based on strain difference influence line $[\mathrm{J}]$. Fujian Construction Science and Technology, 2017(06): 20-22.

3. Guo Xunru. Evaluation of bridge damage using deflection influence line of finite element method [J]. Friends of Science, 2010.

4. Chen Zhiwei, Cai Qinlin. Bridge local damage identification method based on influence line under structural health monitoring system

5. Tang Shenghua, Zhang Jiaqi, Qin Fuqian, et al. Damage identification method of uniform crosssection beam based on the difference of displacement influence line curvature

6. Liu Wenfeng. Application of detection technology and instrument in bridge health monitoring system [M]. Tianjin University Press, 2013

7. Li Guohao. Bridge structure stability and vibration [M]. China Railway Publishing House, 1992.

8. Sun Jue. Health monitoring of bridge structures [J]. Citation Edition: Engineering Technology, 2016, 000(006): P.123-123. 